\title{
Generalizations of Inequalities for Differentiable Co-Ordinated Convex Functions
}

\author{
Feixiang Chen \\ School of Mathematics and Statistics, Chongqing Three Gorges University, Wanzhou, Chongqing 404000, China \\ Correspondence should be addressed to Feixiang Chen; cfx2002@126.com
}

Received 16 November 2013; Accepted 16 January 2014; Published 19 March 2014

Academic Editors: L. Vitagliano and J. Zhang

Copyright (C) 2014 Feixiang Chen. This is an open access article distributed under the Creative Commons Attribution License, which permits unrestricted use, distribution, and reproduction in any medium, provided the original work is properly cited.

A generalized lemmas is proved and several new inequalities for differentiable co-ordinated convex and concave functions in two variables are obtained.

\section{Introduction}

Let $f: I \subseteq \mathbb{R} \rightarrow \mathbb{R}$ be a convex function and $a, b \in I$ with $a<b$; we have the following double inequality:

$$
f\left(\frac{a+b}{2}\right) \leq \frac{1}{b-a} \int_{a}^{b} f(t) d t \leq \frac{f(a)+f(b)}{2} .
$$

This remarkable result is well known in the literature as the Hermite-Hadamard inequality for convex mapping.

Since then, some refinements of the Hermite-Hadamard inequality on convex functions have been extensively investigated by a number of authors (e.g., [1-4]).

A modification for convex functions which is also known as coordinated convex functions was introduced as follows by Dragomir in [5].

Let us consider the bidimensional interval $\Delta:=[a, b] \times$ $[c, d]$ in $\mathbb{R}^{2}$ with $a<b$ and $c<d$; a mapping $f: \Delta \rightarrow \mathbb{R}$ is said to be convex on $\Delta$ if the inequality

$$
\begin{gathered}
f(\lambda x+(1-\lambda) z, \lambda y+(1-\lambda) w) \\
\quad \leq \lambda f(x, y)+(1-\lambda) f(z, w)
\end{gathered}
$$

holds for all $(x, y),(z, w) \in \Delta$, and $\lambda \in[0,1]$.

A function $f: \Delta \rightarrow \mathbb{R}$ is said to be coordinated convex on $\Delta$ if the partial mappings $f_{y}:[a, b] \rightarrow \mathbb{R}, f_{y}(u)=f(u, y)$ and $f_{x}:[c, d] \rightarrow \mathbb{R}, f_{x}(v)=f(x, v)$ are convex for all $y \in$ $[c, d]$ and $x \in[a, b]$.

A formal definition for coordinated convex functions may be stated as follows.
Definition 1. A function $f: \Delta \rightarrow \mathbb{R}$ is said to be convex on coordinates on $\Delta$ if the inequality

$$
\begin{aligned}
f(\lambda x & +(1-\lambda) z, t y+(1-t) w) \\
\leq & \lambda t f(x, y)+\lambda(1-t) f(x, w) \\
& +(1-\lambda) t f(z, y)+(1-t)(1-\lambda) f(z, w)
\end{aligned}
$$

holds for all $(x, y),(z, y),(x, w),(z, w) \in \Delta$, and $t, \lambda \in[0,1]$.

Dragomir in [5] established the following Hadamardtype inequalities for coordinated convex functions in a rectangle from the plane $\mathbb{R}^{2}$.

Theorem 2. Suppose that $f: \Delta=[a, b] \times[c, d] \rightarrow \mathbb{R}$ is convex on the coordinates on $\Delta$. Then one has the inequalities as follows:

$$
\begin{aligned}
& f\left(\frac{a+b}{2}, \frac{c+d}{2}\right) \\
& \quad \leq \frac{1}{(b-a)(d-c)} \int_{a}^{b} \int_{c}^{d} f(x, y) d y d x \\
& \quad \leq \frac{f(a, c)+f(a, d)+f(b, c)+f(b, d)}{4} .
\end{aligned}
$$

Some new integral inequalities that are related to the Hermite-Hadamard type for coordinated convex functions are also established by many authors. 
In ([6], 2008), Alomari and Darus defined coordinated $s$ convex functions and proved some inequalities based on this definition. In ([7], 2009), analogous results for $h$-convex functions on the coordinates were proved by Latif and Alomari. In ([8], 2009), Alomari and Darus established some Hadamardtype inequalities for coordinated log-convex functions.

In ([9], 2012), Latif and Dragomir obtained some new Hadamard type inequalities for differentiable coordinated convex and concave functions in two variables which are related to the left-hand side of Hermite-Hadamard type inequality for coordinated convex functions in two variables based on the following lemma.

Lemma 3. Let $f: \Delta \subseteq \mathbb{R}^{2} \rightarrow \mathbb{R}$ be a partial differentiable mapping on $\Delta:=[a, b] \times[c, d]$ in $\mathbb{R}^{2}$ with $a<b$ and $c<d$. If $\partial^{2} f / \partial u \partial v \in L(\Delta)$, then the following equality holds:

$$
\begin{aligned}
& \frac{1}{(b-a)(d-c)} \int_{a}^{b} \int_{c}^{d} f(x, y) d y d x+f\left(\frac{a+b}{2}, \frac{c+d}{2}\right) \\
& \quad-\frac{1}{b-a} \int_{a}^{b} f\left(x, \frac{c+d}{2}\right) d x \\
& \quad-\frac{1}{d-c} \int_{c}^{d} f\left(\frac{a+b}{2}, y\right) d y \\
& =(b-a)(d-c) \\
& \quad \times \iint_{0}^{1} K(u, v) \frac{\partial^{2} f}{\partial u \partial v}(u a+(1-u) b, v c+(1-v) d) d u d v
\end{aligned}
$$

where

$$
K(u, v)= \begin{cases}u v, & (u, v) \in\left[0, \frac{1}{2}\right] \times\left[0, \frac{1}{2}\right] \\ u(v-1), & (u, v) \in\left[0, \frac{1}{2}\right] \times\left(\frac{1}{2}, 1\right] \\ (u-1) v, & (u, v) \in\left(\frac{1}{2}, 1\right] \times\left[0, \frac{1}{2}\right] \\ (u-1)(v-1), & (u, v) \in\left(\frac{1}{2}, 1\right] \times\left(\frac{1}{2}, 1\right] .\end{cases}
$$

Theorem 4 (see [9]). Let $f: \Delta \subseteq \mathbb{R}^{2} \rightarrow \mathbb{R}$ be a partial differentiable mapping on $\Delta:=[a, b] \times[c, d]$ in $\mathbb{R}^{2}$ with $a<b$ and $c<d$. If $\left|\partial^{2} f / \partial u \partial v\right|$ is convex on the coordinates on $\Delta$, then the following equality holds:

$$
\begin{aligned}
& \left|\frac{1}{(b-a)(d-c)} \int_{a}^{b} \int_{c}^{d} f(x, y) d y d x+f\left(\frac{a+b}{2}, \frac{c+d}{2}\right)-A\right| \\
& \quad \leq \frac{(b-a)(d-c)}{16}
\end{aligned}
$$

$$
\begin{gathered}
\times\left(\left(\left|\frac{\partial^{2} f}{\partial u \partial v}(a, c)\right|+\left|\frac{\partial^{2} f}{\partial u \partial v}(a, d)\right|+\left|\frac{\partial^{2} f}{\partial u \partial v}(b, c)\right|\right.\right. \\
\left.\left.+\left|\frac{\partial^{2} f}{\partial u \partial v}(b, d)\right|\right) \times(4)^{-1}\right),
\end{gathered}
$$

where

$$
A=\frac{1}{b-a} \int_{a}^{b} f\left(x, \frac{c+d}{2}\right) d x+\frac{1}{d-c} \int_{c}^{d} f\left(\frac{a+b}{2}, y\right) d y .
$$

Theorem 5 (see [9]). Let $f: \Delta \subseteq \mathbb{R}^{2} \rightarrow \mathbb{R}$ be a partial differentiable mapping on $\Delta:=[a, b] \times[c, d]$ in $\mathbb{R}^{2}$ with $a<b$ and $c<d$. If $\left|\partial^{2} f / \partial u \partial v\right|^{q}$ is convex on the coordinates on $\Delta$ and $p, q>1,1 / p+1 / q=1$, then the following equality holds:

$$
\begin{gathered}
\left|\frac{1}{(b-a)(d-c)} \int_{a}^{b} \int_{c}^{d} f(x, y) d y d x+f\left(\frac{a+b}{2}, \frac{c+d}{2}\right)-A\right| \\
\leq \frac{(b-a)(d-c)}{4(p+1)^{2 / p}} \\
\times\left(\left(\left|\frac{\partial^{2} f}{\partial u \partial v}(a, c)\right|^{q}+\left|\frac{\partial^{2} f}{\partial u \partial v}(a, d)\right|^{q}+\left|\frac{\partial^{2} f}{\partial u \partial v}(b, c)\right|^{q}\right.\right. \\
\left.\left.+\left|\frac{\partial^{2} f}{\partial u \partial v}(b, d)\right|^{q}\right) \times(4)^{-1}\right)^{1 / q},
\end{gathered}
$$

where $A$ is as given in Theorem 4.

Theorem 6 (see [9]). Let $f: \Delta \subseteq \mathbb{R}^{2} \rightarrow \mathbb{R}$ be a partial differentiable mapping on $\Delta:=[a, b] \times[c, d]$ in $\mathbb{R}^{2}$ with $a<b$ and $c<d$. If $\left|\partial^{2} f / \partial u \partial v\right|^{q}$ is convex on the coordinates on $\Delta$ and $q \geq 1$, then the following equality holds:

$$
\begin{gathered}
\left|\frac{1}{(b-a)(d-c)} \int_{a}^{b} \int_{c}^{d} f(x, y) d y d x+f\left(\frac{a+b}{2}, \frac{c+d}{2}\right)-A\right| \\
\leq \frac{(b-a)(d-c)}{16} \\
\times\left(\left(\left|\frac{\partial^{2} f}{\partial u \partial v}(a, c)\right|^{q}+\left|\frac{\partial^{2} f}{\partial u \partial v}(a, d)\right|^{q}+\left|\frac{\partial^{2} f}{\partial u \partial v}(b, c)\right|^{q}\right.\right. \\
\left.\left.+\left|\frac{\partial^{2} f}{\partial u \partial v}(b, d)\right|^{q}\right) \times(4)^{-1}\right)^{1 / q},
\end{gathered}
$$

where $A$ is as given in Theorem 4.

In ([10], 2012), analogous results which are related to the right-hand side of Hermite-Hadamard type inequality for coordinated convex functions in two variables were proved by Sarkaya et al. based on the following lemma. 
Lemma 7. Let $f: \Delta \subseteq \mathbb{R}^{2} \rightarrow \mathbb{R}$ be a partial differentiable mapping on $\Delta:=[a, b] \times[c, d]$ in $\mathbb{R}^{2}$ with $a<b$ and $c<d$. If $\partial^{2} f / \partial u \partial v \in L(\Delta)$, then the following equality holds:

$$
\begin{aligned}
& \frac{f(a, c)+f(a, d)+f(b, c)+f(b, d)}{4} \\
& +\frac{1}{(b-a)(d-c)} \int_{a}^{b} \int_{c}^{d} f(x, y) d y d x \\
& -\frac{1}{2}\left[\frac{1}{b-a} \int_{a}^{b}[f(x, c)+f(x, d)] d x\right. \\
& \left.\quad+\frac{1}{d-c} \int_{c}^{d}[f(a, y)+f(b, y)] d y\right] \\
& \quad \times \iint_{0}^{1}(1-2 u)(1-2 v) \frac{\partial^{2} f}{\partial u \partial v} \\
& \quad \times(u a+(1-u) b, v c+(1-v) d) d u d v .
\end{aligned}
$$

Theorem 8 (see [10]). Let $f: \Delta \subseteq \mathbb{R}^{2} \rightarrow \mathbb{R}$ be a partial differentiable mapping on $\Delta:=[a, b] \times[c, d]$ in $\mathbb{R}^{2}$ with $a<b$ and $c<d$. If $\left|\partial^{2} f / \partial u \partial v\right|$ is convex on the coordinates on $\Delta$, then the following equality holds:

$$
\begin{aligned}
& \mid \frac{f(a, c)+f(a, d)+f(b, c)+f(b, d)}{4} \\
& +\frac{1}{(b-a)(d-c)} \int_{a}^{b} \int_{c}^{d} f(x, y) d y d x-A \mid \\
& \leq \frac{(b-a)(d-c)}{16} \\
& \quad \times\left(\left(\left|\frac{\partial^{2} f}{\partial u \partial v}(a, c)\right|+\left|\frac{\partial^{2} f}{\partial u \partial v}(a, d)\right|+\left|\frac{\partial^{2} f}{\partial u \partial v}(b, c)\right|\right.\right. \\
& \left.\left.+\left|\frac{\partial^{2} f}{\partial u \partial v}(b, d)\right|\right) \times(4)^{-1}\right),
\end{aligned}
$$

where

$$
\begin{aligned}
A=\frac{1}{2}[ & \frac{1}{b-a} \int_{a}^{b}[f(x, c)+f(x, d)] d x \\
& \left.+\frac{1}{d-c} \int_{c}^{d}[f(a, y)+f(b, y)] d y\right] .
\end{aligned}
$$

Theorem 9 (see [10]). Let $f: \Delta \subseteq \mathbb{R}^{2} \rightarrow \mathbb{R}$ be a partial differentiable mapping on $\Delta:=[a, b] \times[c, d]$ in $\mathbb{R}^{2}$ with $a<b$ and $c<d$. If $\left|\partial^{2} f / \partial u \partial v\right|^{q}$ is convex on the coordinates on $\Delta$ and $p, q>1,1 / p+1 / q=1$, then the following equality holds:

$$
\begin{aligned}
& \mid \frac{f(a, c)+f(a, d)+f(b, c)+f(b, d)}{4} \\
& +\frac{1}{(b-a)(d-c)} \int_{a}^{b} \int_{c}^{d} f(x, y) d y d x-A \mid \\
& \leq \frac{(b-a)(d-c)}{4(p+1)^{2 / p}} \\
& \quad \times\left(\left(\left|\frac{\partial^{2} f}{\partial u \partial v}(a, c)\right|^{q}+\left|\frac{\partial^{2} f}{\partial u \partial v}(a, d)\right|^{q}\right.\right. \\
& \left.\left.\quad+\left|\frac{\partial^{2} f}{\partial u \partial v}(b, c)\right|^{q}+\left|\frac{\partial^{2} f}{\partial u \partial v}(b, d)\right|^{q}\right) \times(4)^{-1}\right)^{1 / q},
\end{aligned}
$$

where $A$ is as given in Theorem 8.

Theorem 10 (see [10]). Let $f: \Delta \subseteq \mathbb{R}^{2} \rightarrow \mathbb{R}$ be a partial differentiable mapping on $\Delta:=[a, b] \times[c, d]$ in $\mathbb{R}^{2}$ with $a<b$ and $c<d$. If $\left|\partial^{2} f / \partial u \partial v\right|^{q}$ is convex on the coordinates on $\Delta$ and $q \geq 1$, then the following equality holds:

$$
\begin{aligned}
& \mid \frac{f(a, c)+f(a, d)+f(b, c)+f(b, d)}{4} \\
& +\frac{1}{(b-a)(d-c)} \int_{a}^{b} \int_{c}^{d} f(x, y) d y d x-A \mid \\
& \leq \frac{(b-a)(d-c)}{16} \\
& \quad \times\left(\left(\left|\frac{\partial^{2} f}{\partial u \partial v}(a, c)\right|^{q}+\left|\frac{\partial^{2} f}{\partial u \partial v}(a, d)\right|^{q}\right.\right. \\
& \left.\left.\quad+\left|\frac{\partial^{2} f}{\partial u \partial v}(b, c)\right|^{q}+\left|\frac{\partial^{2} f}{\partial u \partial v}(b, d)\right|^{q}\right) \times(4)^{-1}\right)^{1 / q},
\end{aligned}
$$

where $A$ is as given in Theorem 8.

In [11], Ozdemir et al. established some Simpson's inequalities for coordinated convex functions based on the following lemma.

Lemma 11. Let $f: \Delta \subseteq \mathbb{R}^{2} \rightarrow \mathbb{R}$ be a partial differentiable mapping on $\Delta:=[a, b] \times[c, d]$ in $\mathbb{R}^{2}$ with $a<b$ and $c<d$. If $\partial^{2} f / \partial u \partial v \in L(\Delta)$, then the following equality holds:

$$
\begin{gathered}
\left(f\left(a, \frac{c+d}{2}\right)+f\left(b, \frac{c+d}{2}\right)+4 f\left(\frac{a+b}{2}, \frac{c+d}{2}\right)\right. \\
\left.+f\left(\frac{a+b}{2}, c\right)+f\left(\frac{a+b}{2}, d\right) \times(9)^{-1}\right)
\end{gathered}
$$




$$
\begin{aligned}
& +\frac{f(a, c)+f(a, d)+f(b, c)+f(b, d)}{36} \\
& -\frac{1}{6(b-a)} \int_{a}^{b}\left(f(x, c)+4 f\left(x, \frac{c+d}{2}\right)+f(x, d)\right) d x \\
& -\frac{1}{6(d-c)} \int_{c}^{d}\left(f(a, y)+4 f\left(\frac{a+b}{2}, y\right)+f(b, y)\right) d y \\
& +\frac{1}{(b-a)(d-c)} \int_{a}^{b} \int_{c}^{d} f(x, y) d y d x \\
& =(b-a)(d-c) \\
& \quad \times \iint_{0}^{1} p(x, u) p(y, v) \frac{\partial^{2} f}{\partial u \partial v} \\
& \quad \times(u a+(1-u) b, v c+(1-v) d) d u d v
\end{aligned}
$$

where

$$
\begin{aligned}
& p(x, u)= \begin{cases}\left(u-\frac{1}{6}\right), & t \in\left[0, \frac{1}{2}\right] \\
\left(u-\frac{5}{6}\right), & t \in\left(\frac{1}{2}, 1\right],\end{cases} \\
& p(y, v)= \begin{cases}\left(v-\frac{1}{6}\right), & s \in\left[0, \frac{1}{2}\right] \\
\left(v-\frac{5}{6}\right), & s \in\left(\frac{1}{2}, 1\right] .\end{cases}
\end{aligned}
$$

Theorem 12 (see [11]). Let $f: \Delta \subseteq \mathbb{R}^{2} \rightarrow \mathbb{R}$ be a partial differentiable mapping on $\Delta:=[a, b] \times[c, d]$. If $\left|\partial^{2} f / \partial u \partial v\right|$ is convex on the coordinates on $\Delta$, then the following equality holds:

$$
\begin{aligned}
& \mid\left(f\left(a, \frac{c+d}{2}\right)+f\left(b, \frac{c+d}{2}\right)+4 f\left(\frac{a+b}{2}, \frac{c+d}{2}\right)\right. \\
&+\left.f\left(\frac{a+b}{2}, c\right)+f\left(\frac{a+b}{2}, d\right) \times(9)^{-1}\right) \\
&+\frac{f(a, c)+f(a, d)+f(b, c)+f(b, d)}{36} \\
&+\frac{1}{(b-a)(d-c)} \int_{a}^{b} \int_{c}^{d} f(x, y) d y d x-A \mid \\
& \leq\left(\frac{5}{72}\right)^{2}(b-a)(d-c) \\
& \times\left(\left|\frac{\partial^{2} f}{\partial u \partial v}(a, c)\right|+\left|\frac{\partial^{2} f}{\partial u \partial v}(a, d)\right|+\left|\frac{\partial^{2} f}{\partial u \partial v}(b, c)\right|\right. \\
&\left.+\left|\frac{\partial^{2} f}{\partial u \partial v}(b, d)\right|\right)
\end{aligned}
$$

where

$$
\begin{aligned}
A= & \frac{1}{6(b-a)} \int_{a}^{b}\left(f(x, c)+4 f\left(x, \frac{c+d}{2}\right)+f(x, d)\right) d x \\
& +\frac{1}{6(d-c)} \int_{c}^{d}\left(f(a, y)+4 f\left(\frac{a+b}{2}, y\right)+f(b, y)\right) d y .
\end{aligned}
$$

For recent results and generalizations concerning Hermite-Hadamard type inequality for differentiable coordinated convex functions see $([12], 2012)$ and the references given therein.

In this paper, a generalized lemma is proved and several new inequalities for differentiable coordinated convex and concave functions in two variables are obtained.

\section{Lemmas}

To establish our results, we need the following lemma.

Lemma 13. Let $f: \Delta \subseteq \mathbb{R}^{2} \rightarrow \mathbb{R}$ be a partial differentiable mapping on $\Delta:=[a, b] \times[c, d]$ in $\mathbb{R}^{2}$ with $a<b$ and $c<d$. If $\partial^{2} f / \partial u \partial v \in L(\Delta)$ and $\lambda \in[0,1]$, then the following equality holds:

$$
\begin{aligned}
& \frac{1}{(b-a)(d-c)} \int_{a}^{b} \int_{c}^{d} f(x, y) d y d x \\
& +(1-\lambda)^{2} f\left(\frac{a+b}{2}, \frac{c+d}{2}\right)+\frac{\lambda(1-\lambda)}{2} \\
& \times\left[f\left(a, \frac{c+d}{2}\right)+f\left(a, \frac{c+d}{2}\right)\right. \\
& +\frac{\lambda^{2}}{4}[f(a, c)+f(a, d)+f(b, c)+f(b, d)] \\
& \left.\quad-\frac{1}{2(b-a)}, f\left(\frac{a+b}{2}, c\right)+f\left(\frac{a+b}{2}, d\right)\right] \\
& \quad \times \int_{a}^{b}\left(\lambda f(x, c)+2(1-\lambda) f\left(x, \frac{c+d}{2}\right)+\lambda f(x, d)\right) d x \\
& \quad \times \quad \frac{1}{2(d-c)}(\lambda-a)(d-c) \\
& \quad \int_{c}^{d}\left(u f(a, y)+2(1-\lambda) f\left(\frac{a+b}{2}, y\right)+\lambda f(b, y)\right) d y
\end{aligned}
$$


where

$$
M(u, v)=\left\{\begin{array}{c}
\left(u-\frac{\lambda}{2}\right)\left(v-\frac{\lambda}{2}\right), \\
(u, v) \in\left[0, \frac{1}{2}\right] \times\left[0, \frac{1}{2}\right] \\
\left(u-\frac{\lambda}{2}\right)\left(v-\left(1-\frac{\lambda}{2}\right)\right), \\
(u, v) \in\left[0, \frac{1}{2}\right] \times\left(\frac{1}{2}, 1\right] \\
\left(u-\left(1-\frac{\lambda}{2}\right)\right)\left(v-\frac{\lambda}{2}\right) \\
(u, v) \in\left(\frac{1}{2}, 1\right] \times\left[0, \frac{1}{2}\right] \\
\left(u-\left(1-\frac{\lambda}{2}\right)\right)\left(v-\left(1-\frac{\lambda}{2}\right)\right) \\
(u, v) \in\left(\frac{1}{2}, 1\right] \times\left(\frac{1}{2}, 1\right] .
\end{array}\right.
$$

Proof. Since

$$
\begin{gathered}
\iint_{0}^{1} M(u, v) \frac{\partial^{2} f}{\partial u \partial v} \\
\quad \times(u a+(1-u) b, v c+(1-v) d) d u d v \\
=\iint_{0}^{1 / 2}\left(u-\frac{\lambda}{2}\right)\left(v-\frac{\lambda}{2}\right) \frac{\partial^{2} f}{\partial u \partial v} \\
\quad \times(u a+(1-u) b, v c+(1-v) d) d u d v \\
+\int_{0}^{1 / 2} \int_{1 / 2}^{1}\left(u-\frac{\lambda}{2}\right)\left(v-\left(1-\frac{\lambda}{2}\right)\right) \frac{\partial^{2} f}{\partial u \partial v} \\
\quad \times(u a+(1-u) b, v c+(1-v) d) d u d v \\
+\int_{1 / 2}^{1} \int_{0}^{1 / 2}\left(u-\left(1-\frac{\lambda}{2}\right)\right)\left(v-\frac{\lambda}{2}\right) \frac{\partial^{2} f}{\partial u \partial v} \\
\quad \times(u a+(1-u) b, v c+(1-v) d) d u d v \\
+\iint_{1 / 2}^{1}\left(u-\left(1-\frac{\lambda}{2}\right)\right)\left(v-\left(1-\frac{\lambda}{2}\right)\right) \frac{\partial^{2} f}{\partial u \partial v} \\
\quad \times(u a+(1-u) b, v c+(1-v) d) d u d v
\end{gathered}
$$

$$
\begin{aligned}
& \times(u a+(1-u) b, v c+(1-v) d) d v\} d u \\
& =\frac{1-\lambda}{2} \frac{1}{c-d} \int_{0}^{1 / 2}\left(u-\frac{\lambda}{2}\right) \frac{\partial f}{\partial u}\left(u a+(1-u) b, \frac{c+d}{2}\right) d u \\
& +\frac{\lambda}{2} \frac{1}{c-d} \int_{0}^{1 / 2}\left(u-\frac{\lambda}{2}\right) \frac{\partial f}{\partial u}(u a+(1-u) b, d) d u \\
& -\frac{1}{c-d} \iint_{0}^{1 / 2}\left(u-\frac{\lambda}{2}\right) \frac{\partial f}{\partial u} \\
& \times(u a+(1-u) b, v c+(1-v) d) d v d u \\
& =\frac{1-\lambda}{2} \frac{1}{c-d} \frac{1}{a-b}\left(u-\frac{\lambda}{2}\right) \\
& \times\left. f\left(u a+(1-u) b, \frac{c+d}{2}\right)\right|_{0} ^{1 / 2} \\
& -\frac{1-\lambda}{2} \frac{1}{c-d} \frac{1}{a-b} \int_{0}^{1 / 2} f\left(u a+(1-u) b, \frac{c+d}{2}\right) d u \\
& +\left.\frac{\lambda}{2} \frac{1}{c-d} \frac{1}{a-b}\left(u-\frac{\lambda}{2}\right) f(u a+(1-u) b, d)\right|_{0} ^{1 / 2} \\
& -\frac{\lambda}{2} \frac{1}{c-d} \frac{1}{a-b} \int_{0}^{1 / 2} f(u a+(1-u) b, d) d u \\
& -\frac{1}{c-d} \frac{1}{a-b} \\
& \times \int_{0}^{1 / 2}\left\{\left.\left(u-\frac{\lambda}{2}\right) f(u a+(1-u) b, v c+(1-v) d)\right|_{0} ^{1 / 2}\right. \\
& \left.-\int_{0}^{1 / 2} f(u a+(1-u) b, v c+(1-v) d) d u\right\} d v \\
& =\frac{(1-\lambda)^{2}}{4(c-d)(a-b)} f\left(\frac{a+b}{2}, \frac{c+d}{2}\right) \\
& +\frac{\lambda(1-\lambda)}{4(c-d)(a-b)} f\left(b, \frac{c+d}{2}\right) \\
& -\frac{1-\lambda}{2(c-d)(a-b)} \int_{0}^{1 / 2} f\left(u a+(1-u) b, \frac{c+d}{2}\right) d u \\
& +\frac{\lambda(1-\lambda)}{4(c-d)(a-b)} f\left(\frac{a+b}{2}, d\right) \\
& +\frac{\lambda^{2}}{4(c-d)(a-b)} f(b, d)
\end{aligned}
$$




$$
\begin{aligned}
& -\frac{\lambda}{2(c-d)(a-b)} \int_{0}^{1 / 2} f(u a+(1-u) b, d) d u \\
& -\frac{1-\lambda}{2(c-d)(a-b)} \int_{0}^{1 / 2} f\left(\frac{a+b}{2}, v c+(1-v) d\right) d v \\
& -\frac{\lambda}{2(c-d)(a-b)} \int_{0}^{1 / 2} f(b, v c+(1-v) d) d v \\
& +\frac{1}{(c-d)(a-b)} \\
& \times \iint_{0}^{1 / 2} f(u a+(1-u) b, v c+(1-v) d) d u d v
\end{aligned}
$$

Similarly, we can get

$$
\begin{aligned}
\int_{0}^{1 / 2} \int_{1 / 2}^{1}\left(u-\frac{\lambda}{2}\right)\left(v-\left(1-\frac{\lambda}{2}\right)\right) \frac{\partial^{2} f}{\partial u \partial v} \\
\quad \times(u a+(1-u) b, v c+(1-v) d) d u d v \\
=\frac{(1-\lambda)^{2}}{4(c-d)(a-b)} f\left(\frac{a+b}{2}, \frac{c+d}{2}\right) \\
+\frac{\lambda(1-\lambda)}{4(c-d)(a-b)} f\left(b, \frac{c+d}{2}\right)-\frac{1-\lambda}{2(c-d)(a-b)} \\
\quad \times \int_{0}^{1 / 2} f\left(u a+(1-u) b, \frac{c+d}{2}\right) d u \\
\quad+\frac{\lambda(1-\lambda)}{4(c-d)(a-b)} f\left(\frac{a+b}{2}, c\right) \\
\quad+\frac{\lambda^{2}}{4(c-d)(a-b)} f(b, c)-\frac{\lambda}{2(c-d)(a-b)} \\
\quad \times \int_{1 / 2}^{1} \int_{0}^{1 / 2} \int_{1 / 2}^{1 / 2} f(u a+(1-u) b, v c+(1-v) d) d u d v \\
\quad \times \int_{0}^{1 / 2} f(u a+(1-u) b, c) d u \\
\quad-\frac{1-\lambda}{2(c-d)(a-b)} \\
\quad \int_{1 / 2}^{1} f\left(\frac{a+b}{2}, v c+(1-v) d\right) d v-\frac{\lambda}{2(c-d)(b-a)} \\
(c-d)(a-b)
\end{aligned}
$$

$$
\begin{aligned}
= & \frac{(1-\lambda)^{2}}{4(c-d)(a-b)} f\left(\frac{a+b}{2}, \frac{c+d}{2}\right) \\
& +\frac{\lambda(1-\lambda)}{4(c-d)(a-b)} f\left(a, \frac{c+d}{2}\right) \\
& -\frac{1-\lambda}{2(c-d)(a-b)} \int_{1 / 2}^{1} f\left(u a+(1-u) b, \frac{c+d}{2}\right) d u \\
& +\frac{\lambda(1-\lambda)}{4(c-d)(a-b)} f\left(\frac{a+b}{2}, d\right) \\
& +\frac{\lambda^{2}}{4(d-c)(b-a)} f(a, d)-\frac{\lambda}{2(c-d)(a-b)} \\
& \times \int_{1 / 2}^{1} f(u a+(1-u) b, d) d u
\end{aligned}
$$$$
-\frac{1-\lambda}{2(c-d)(a-b)}
$$$$
\times \int_{0}^{1 / 2} f\left(\frac{a+b}{2}, v c+(1-v) d\right) d v
$$$$
-\frac{\lambda}{2(d-c)(b-a)}
$$$$
\times \int_{0}^{1 / 2} f(a, v c+(1-v) d) d v+\frac{1}{(c-d)(a-b)}
$$$$
\times \int_{1 / 2}^{1} \int_{0}^{1 / 2} f(u a+(1-u) b, v c+(1-v) d) d u d v
$$$$
\iint_{1 / 2}^{1}\left(u-\left(1-\frac{\lambda}{2}\right)\right)\left(v-\left(1-\frac{\lambda}{2}\right)\right) \frac{\partial^{2} f}{\partial u \partial v}
$$$$
\times(u a+(1-u) b, v c+(1-v) d) d u d v
$$$$
=\frac{(1-\lambda)^{2}}{4(c-d)(a-b)} f\left(\frac{a+b}{2}, \frac{c+d}{2}\right)
$$$$
+\frac{\lambda(1-\lambda)}{4(c-d)(a-b)} f\left(a, \frac{c+d}{2}\right)
$$$$
-\frac{1-\lambda}{2(c-d)(a-b)} \int_{1 / 2}^{1} f\left(u a+(1-u) b, \frac{c+d}{2}\right) d u
$$$$
+\frac{\lambda(1-\lambda)}{4(c-d)(a-b)} f\left(\frac{a+b}{2}, c\right)
$$$$
+\frac{\lambda^{2}}{4(c-d)(a-b)} f(a, c)-\frac{\lambda}{2(c-d)(a-b)}
$$$$
\times \int_{1 / 2}^{1} f(u a+(1-u) b, c) d u
$$$$
-\frac{1-\lambda}{2(c-d)(a-b)}
$$$$
\times \int_{1 / 2}^{1} f\left(\frac{a+b}{2}, v c+(1-v) d\right) d v
$$ 


$$
\begin{aligned}
& -\frac{\lambda}{2(c-d)(a-b)} \int_{1 / 2}^{1} f(a, v c+(1-v) d) d v \\
& +\frac{1}{(c-d)(a-b)} \\
& \times \iint_{1 / 2}^{1} f(u a+(1-u) b, v c+(1-v) d) d u d v .
\end{aligned}
$$

Now

$$
\begin{aligned}
\iint_{0}^{1} M & (u, v) \frac{\partial^{2} f}{\partial u \partial v}(u a+(1-u) b, v c+(1-v) d) d u d v \\
= & \frac{(1-\lambda)^{2}}{(d-c)(b-a)} f\left(\frac{a+b}{2}, \frac{c+d}{2}\right)+\frac{\lambda(1-\lambda)}{2(d-c)(b-a)} \\
& \times\left[f\left(a, \frac{c+d}{2}\right)+f\left(b, \frac{c+d}{2}\right)\right. \\
& +\frac{\lambda^{2}}{4(d-c)(b-a)} \\
& \times[f(a, c)+f(b, c)+f(a, d)+f(b, d)] \\
& +\frac{1}{2(d-c)(b-a)}, \frac{\partial^{2} f}{2 u \partial v}(u a+(1-u) b, v c+(1-v) d) d u d v \\
\quad & +\frac{1}{2(d-c)(b-a)} \\
\quad & +\int_{0}^{1}[\lambda f(u a+(1-u) b, c) \\
& +2(1-\lambda) f\left(u a+(1-u) b, \frac{c+d}{2}\right)
\end{aligned}
$$

Multiplying both sides by $(b-a)(d-c)$ and using the change of the variable $x=u a+(1-u) b$ and $y=v c+(1-v) d$, which completes the proof.
Remark 14. Applying Lemma 13 for $\lambda=0,1,1 / 3$, we get the results of Lemmas 3, 7, and 11, respectively.

\section{Main Results}

Theorem 15. Let $f: \Delta \subseteq \mathbb{R}^{2} \rightarrow \mathbb{R}$ be a partial differentiable mapping on $\Delta:=[a, b] \times[c, d]$ in $\mathbb{R}^{2}$ with $a<b$ and $c<d$. If $\left|\partial^{2} f / \partial u \partial v\right|$ is convex on the coordinates on $\Delta$ and $\lambda \in[0,1]$, then the following equality holds:

$$
\begin{aligned}
& \mid \frac{1}{(b-a)(d-c)} \int_{a}^{b} \int_{c}^{d} f(x, y) d y d x \\
& +(1-\lambda)^{2} f\left(\frac{a+b}{2}, \frac{c+d}{2}\right) \\
& +\frac{\lambda^{2}}{4}[f(a, c)+f(a, d)+f(b, c)+f(b, d)]-A \mid \\
& \leq(b-a)(d-c)\left(\frac{2 \lambda^{2}-2 \lambda+1}{8}\right)^{2} \\
& \times\left(\left|\frac{\partial^{2} f}{\partial u \partial v}(a, c)\right|+\left|\frac{\partial^{2} f}{\partial u \partial v}(a, d)\right|\right. \\
& \left.+\left|\frac{\partial^{2} f}{\partial u \partial v}(b, c)\right|+\left|\frac{\partial^{2} f}{\partial u \partial v}(b, d)\right|\right)
\end{aligned}
$$

where

$$
\begin{aligned}
A= & \frac{1}{2(b-a)} \\
& \times \int_{a}^{b}\left(\lambda f(x, c)+2(1-\lambda) f\left(x, \frac{c+d}{2}\right)+\lambda f(x, d)\right) d x \\
& +\frac{1}{2(d-c)} \\
& \times \int_{c}^{d}\left(\lambda f(a, y)+2(1-\lambda) f\left(\frac{a+b}{2}, y\right)+\lambda f(b, y)\right) d y \\
& -\frac{\lambda(1-\lambda)}{2} \\
& \times\left[f\left(a, \frac{c+d}{2}\right)+f\left(a, \frac{c+d}{2}\right)\right. \\
& \left.+f\left(\frac{a+b}{2}, c\right)+f\left(\frac{a+b}{2}, d\right)\right] .
\end{aligned}
$$

Proof. From Lemma 13, we obtain

$$
\begin{aligned}
& \mid \frac{1}{(b-a)(d-c)} \int_{a}^{b} \int_{c}^{d} f(x, y) d y d x \\
& +(1-\lambda)^{2} f\left(\frac{a+b}{2}, \frac{c+d}{2}\right)
\end{aligned}
$$




$$
\begin{aligned}
& +\frac{\lambda^{2}}{4}[f(a, c)+f(a, d)+f(b, c)+f(b, d)]-A \mid \\
& \leq(b-a)(d-c) \\
& \quad \times \iint_{0}^{1}|M(u, v)| \\
& \quad \times\left|\frac{\partial^{2} f}{\partial u \partial v}(u a+(1-u) b, v c+(1-v) d)\right| d u d v .
\end{aligned}
$$

Because $\left|\partial^{2} f / \partial u \partial v\right|$ is a convex function on the coordinates on $\Delta$, then one has

$$
\begin{aligned}
& \mid \frac{1}{(b-a)(d-c)} \int_{a}^{b} \int_{c}^{d} f(x, y) d y d x \\
& +(1-\lambda)^{2} f\left(\frac{a+b}{2}, \frac{c+d}{2}\right) \\
& +\frac{\lambda^{2}}{4}[f(a, c)+f(a, d)+f(b, c)+f(b, d)]-A \\
& \leq(b-a)(d-c) \\
& \times \iint_{0}^{1}|M(u, v)| \\
& \times\left\{u v\left|\frac{\partial^{2} f}{\partial u \partial v}(a, c)\right|+u(1-v)\left|\frac{\partial^{2} f}{\partial u \partial v}(a, d)\right|\right. \\
& +(1-u) v\left|\frac{\partial^{2} f}{\partial u \partial v}(b, c)\right| \\
& \left.+(1-u)(1-v)\left|\frac{\partial^{2} f}{\partial u \partial v}(b, d)\right|\right\} d u d v \\
& =(b-a)(d-c) \\
& \times\left\{\left|\frac{\partial^{2} f}{\partial u \partial v}(a, c)\right| \iint_{0}^{1}|M(u, v)| u v d u d v\right. \\
& +\left|\frac{\partial^{2} f}{\partial u \partial v}(a, d)\right| \iint_{0}^{1}|M(u, v)| u(1-v) d u d v \\
& +\left|\frac{\partial^{2} f}{\partial u \partial v}(b, c)\right| \iint_{0}^{1}|M(u, v)|(1-u) v d u d v \\
& \left.+\left|\frac{\partial^{2} f}{\partial u \partial v}(b, d)\right| \iint_{0}^{1}|M(u, v)|(1-u)(1-v) d u d v\right\} \\
& =(b-a)(d-c)\left(\frac{2 \lambda^{2}-2 \lambda+1}{8}\right)^{2}
\end{aligned}
$$

$$
\begin{aligned}
& \times\left(\left|\frac{\partial^{2} f}{\partial u \partial v}(a, c)\right|+\left|\frac{\partial^{2} f}{\partial u \partial v}(a, d)\right|+\left|\frac{\partial^{2} f}{\partial u \partial v}(b, c)\right|\right. \\
& \left.+\left|\frac{\partial^{2} f}{\partial u \partial v}(b, d)\right|\right) .
\end{aligned}
$$

On the other hand, we have

$$
\begin{aligned}
& \iint_{0}^{1}|M(u, v)| u v d u d v \\
& =\iint_{0}^{1}|M(u, v)| u(1-v) d u d v \\
& =\iint_{0}^{1}|M(u, v)|(1-u) v d u d v \\
& =\iint_{0}^{1}|M(u, v)|(1-u)(1-v) d u d v, \\
& \iint_{0}^{1}|M(u, v)| u v d u d v \\
& =\iint_{0}^{1 / 2}\left|\left(u-\frac{\lambda}{2}\right)\left(v-\frac{\lambda}{2}\right)\right| u v d u d v \\
& +\int_{0}^{1 / 2} \int_{1 / 2}^{1}\left|\left(u-\frac{\lambda}{2}\right)\left(v-\left(1-\frac{\lambda}{2}\right)\right)\right| u v d u d v \\
& +\int_{1 / 2}^{1} \int_{0}^{1 / 2}\left|\left(u-\left(1-\frac{\lambda}{2}\right)\right)\left(v-\frac{\lambda}{2}\right)\right| u v d u d v \\
& +\iint_{1 / 2}^{1}\left|\left(u-\left(1-\frac{\lambda}{2}\right)\right)\left(v-\left(1-\frac{\lambda}{2}\right)\right)\right| u v d u d v \\
& =\left(\frac{2 \lambda^{2}-2 \lambda+1}{8}\right)^{2} \text {, }
\end{aligned}
$$

which completes the proof.

Remark 16. Applying Theorem 15 for $\lambda=0,1,1 / 3$, we get the results of Theorems 4,8 , and 12 , respectively.

Theorem 17. Let $f: \Delta \subseteq \mathbb{R}^{2} \rightarrow \mathbb{R}$ be a partial differentiable mapping on $\Delta:=[a, b] \times[c, d]$ in $\mathbb{R}^{2}$ with $a<b$ and $c<d$. If $\left|\partial^{2} f / \partial u \partial v\right|^{q}$ is convex on the coordinates on $\Delta$ and $q>1$, one gets the following inequality:

$$
\begin{aligned}
& \mid \frac{1}{(b-a)(d-c)} \int_{a}^{b} \int_{c}^{d} f(x, y) d y d x \\
& +(1-\lambda)^{2} f\left(\frac{a+b}{2}, \frac{c+d}{2}\right) \\
& +\frac{\lambda^{2}}{4}[f(a, c)+f(a, d)+f(b, c)+f(b, d)]-A \mid \\
& \quad \leq \frac{(b-a)(d-c)}{4}\left(\frac{\lambda^{p+1}+(1-\lambda)^{p+1}}{p+1}\right)^{2 / p}
\end{aligned}
$$




$$
\begin{aligned}
& \times\left(\left(\left|\frac{\partial^{2} f}{\partial u \partial v}(a, c)\right|^{q}+\left|\frac{\partial^{2} f}{\partial u \partial v}(a, d)\right|^{q}\right.\right. \\
& \left.\left.+\left|\frac{\partial^{2} f}{\partial u \partial v}(b, c)\right|^{q}+\left|\frac{\partial^{2} f}{\partial u \partial v}(b, d)\right|^{q}\right) \times(4)^{-1}\right)^{1 / q},
\end{aligned}
$$

where $\lambda \in[0,1]$ and $A$ is as given in Theorem 15 and $1 / p+$ $1 / q=1$.

Proof. From Lemma 13, we obtain

$$
\begin{aligned}
& \mid \frac{1}{(b-a)(d-c)} \int_{a}^{b} \int_{c}^{d} f(x, y) d y d x \\
& +(1-\lambda)^{2} f\left(\frac{a+b}{2}, \frac{c+d}{2}\right) \\
& +\frac{\lambda^{2}}{4}[f(a, c)+f(a, d)+f(b, c)+f(b, d)]-A \mid \\
& \leq(b-a)(d-c) \\
& \quad \times \iint_{0}^{1}|M(u, v)| \\
& \quad \times\left|\frac{\partial^{2} f}{\partial u \partial v}(u a+(1-u) b, v c+(1-v) d)\right| d u d v .
\end{aligned}
$$

By using the well-known Hölder inequality for double integrals, then one has

$$
\begin{aligned}
& \mid \frac{1}{(b-a)(d-c)} \int_{a}^{b} \int_{c}^{d} f(x, y) d y d x \\
& +(1-\lambda)^{2} f\left(\frac{a+b}{2}, \frac{c+d}{2}\right) \\
& +\frac{\lambda^{2}}{4}[f(a, c)+f(a, d)+f(b, c)+f(b, d)]-A \mid \\
& \leq(b-a)(d-c)\left(\iint_{0}^{1}|M(u, v)|^{p} d u d v\right)^{1 / p} \\
& \quad \times\left(\iint_{0}^{1} \mid \frac{\partial^{2} f}{\partial u \partial v}(u a+(1-u) b, v c\right. \\
& \left.\quad+(1-v) d)\left.\right|^{q} d u d v\right)^{1 / q} .
\end{aligned}
$$

Because $\left|\partial^{2} f / \partial u \partial v\right|^{q}$ is a convex function on the coordinates on $\Delta$, by (4), then one has

$$
\iint_{0}^{1}\left|\frac{\partial^{2} f}{\partial u \partial v}(u a+(1-u) b, v c+(1-v) d)\right|^{q} d u d v
$$

$$
\begin{gathered}
\leq\left(\left(\left|\frac{\partial^{2} f}{\partial u \partial v}(a, c)\right|^{q}+\left|\frac{\partial^{2} f}{\partial u \partial v}(a, d)\right|^{q}+\left|\frac{\partial^{2} f}{\partial u \partial v}(b, c)\right|^{q}\right.\right. \\
\left.\left.+\left|\frac{\partial^{2} f}{\partial u \partial v}(b, d)\right|^{q}\right) \times(4)^{-1}\right) .
\end{gathered}
$$

We note that

$$
\begin{aligned}
& \iint_{0}^{1}|M(u, v)|^{p} d u d v \\
& \quad=\iint_{0}^{1 / 2}\left|\left(u-\frac{\lambda}{2}\right)\left(v-\frac{\lambda}{2}\right)\right|^{p} d u d v \\
& \quad+\int_{0}^{1 / 2} \int_{1 / 2}^{1}\left|\left(u-\frac{\lambda}{2}\right)\left(v-\left(1-\frac{\lambda}{2}\right)\right)\right|^{p} d u d v \\
& \quad+\int_{1 / 2}^{1} \int_{0}^{1 / 2}\left|\left(u-\left(1-\frac{\lambda}{2}\right)\right)\left(v-\frac{\lambda}{2}\right)\right|^{p} d u d v \\
& \quad+\iint_{1 / 2}^{1}\left|\left(u-\left(1-\frac{\lambda}{2}\right)\right)\left(v-\left(1-\frac{\lambda}{2}\right)\right)\right|^{p} d u d v \\
& \quad=\left(\frac{\lambda^{p+1}+(1-\lambda)^{p+1}}{(p+1) 2^{p}}\right) .
\end{aligned}
$$

Hence, it follows that

$$
\begin{aligned}
& \mid \frac{1}{(b-a)(d-c)} \int_{a}^{b} \int_{c}^{d} f(x, y) d y d x \\
& +(1-\lambda)^{2} f\left(\frac{a+b}{2}, \frac{c+d}{2}\right) \\
& +\frac{\lambda^{2}}{4}[f(a, c)+f(a, d)+f(b, c)+f(b, d)]-A \mid \\
& \leq \frac{(b-a)(d-c)}{4}\left(\frac{\lambda^{p+1}+(1-\lambda)^{p+1}}{p+1}\right)^{2 / p} \\
& \quad \times\left(\left(\left|\frac{\partial^{2} f}{\partial u \partial v}(a, c)\right|^{q}+\left|\frac{\partial^{2} f}{\partial u \partial v}(a, d)\right|^{q}+\left|\frac{\partial^{2} f}{\partial u \partial v}(b, c)\right|^{q}\right.\right. \\
& \left.\left.\quad+\left|\frac{\partial^{2} f}{\partial u \partial v}(b, d)\right|^{q}\right) \times(4)^{-1}\right)^{1 / q} \cdot
\end{aligned}
$$

Remark 18. Applying Theorem 17 for $\lambda=0$, 1, we get the results of Theorems 5 and 9 , respectively.

Theorem 19. Let $f: \Delta \subseteq \mathbb{R}^{2} \rightarrow \mathbb{R}$ be a partial differentiable mapping on $\Delta:=[a, b] \times[c, d]$ in $\mathbb{R}^{2}$ with $a<b$ and $c<d$. If $\left.\left|\partial^{2} f / \partial u \partial v\right|\right|^{q}$ is convex on the coordinates on $\Delta$ and $q \geq 1$, then

$$
\begin{aligned}
& \mid \frac{1}{(b-a)(d-c)} \int_{a}^{b} \int_{c}^{d} f(x, y) d y d x \\
& +(1-\lambda)^{2} f\left(\frac{a+b}{2}, \frac{c+d}{2}\right)
\end{aligned}
$$




$$
\begin{aligned}
& +\frac{\lambda^{2}}{4}[f(a, c)+f(a, d)+f(b, c)+f(b, d)]-A \mid \\
& \leq(b-a)(d-c)\left(\frac{2 \lambda^{2}-2 \lambda+1}{4}\right)^{2} \\
& \times\left(\left(\left|\frac{\partial^{2} f}{\partial u \partial v}(a, c)\right|^{q}+\left|\frac{\partial^{2} f}{\partial u \partial v}(a, d)\right|^{q}+\left|\frac{\partial^{2} f}{\partial u \partial v}(b, c)\right|^{q}\right.\right. \\
& \left.\left.+\left|\frac{\partial^{2} f}{\partial u \partial v}(b, d)\right|^{q}\right) \times(4)^{-1}\right)^{1 / q},
\end{aligned}
$$

where $\lambda \in[0,1]$ and $A$ is as given in Theorem 15 .

Proof. From Lemma 13, we obtain

$$
\begin{aligned}
& \mid \frac{1}{(b-a)(d-c)} \int_{a}^{b} \int_{c}^{d} f(x, y) d y d x \\
& +(1-\lambda)^{2} f\left(\frac{a+b}{2}, \frac{c+d}{2}\right) \\
& +\frac{\lambda^{2}}{4}[f(a, c)+f(a, d)+f(b, c)+f(b, d)]-A \mid \\
& \leq(b-a)(d-c) \\
& \quad \times \iint_{0}^{1}|M(u, v)| \\
& \quad \times\left|\frac{\partial^{2} f}{\partial u \partial v}(u a+(1-u) b, v c+(1-v) d)\right| d u d v
\end{aligned}
$$

By using the well-known power mean inequality for double integrals, then one has

$$
\begin{aligned}
& \mid \frac{1}{(b-a)(d-c)} \int_{a}^{b} \int_{c}^{d} f(x, y) d y d x \\
& +(1-\lambda)^{2} f\left(\frac{a+b}{2}, \frac{c+d}{2}\right) \\
& +\frac{\lambda^{2}}{4}[f(a, c)+f(a, d)+f(b, c)+f(b, d)]-A \mid \\
& \leq(b-a)(d-c)\left(\iint_{0}^{1}|M(u, v)| d u d v\right)^{1-(1 / q)} \\
& \quad \times\left(\iint_{0}^{1}|M(u, v)|\right.
\end{aligned}
$$

$$
\begin{aligned}
\times \mid \frac{\partial^{2} f}{\partial u \partial v}(u a & +(1-u) b, v c \\
& \left.+(1-v) d)\left.\right|^{q} d u d v\right)^{1 / q}
\end{aligned}
$$

Because $\left|\partial^{2} f / \partial u \partial v\right|^{q}$ is a convex function on the coordinates on $\Delta$, then one has

$$
\begin{aligned}
& \left|\frac{\partial^{2} f}{\partial u \partial v}(u a+(1-u) b, v c+(1-v) d)\right|^{q} \\
& \leq u v\left|\frac{\partial^{2} f}{\partial u \partial v}(a, c)\right|^{q}+u(1-v)\left|\frac{\partial^{2} f}{\partial u \partial v}(a, d)\right|^{q} \\
& \quad+(1-u) v\left|\frac{\partial^{2} f}{\partial u \partial v}(b, c)\right|^{q} \\
& \quad+(1-u)(1-v)\left|\frac{\partial^{2} f}{\partial u \partial v}(b, d)\right|^{q} .
\end{aligned}
$$

Thus, it follows that

$$
\begin{aligned}
& \mid \frac{1}{(b-a)(d-c)} \int_{a}^{b} \int_{c}^{d} f(x, y) d y d x \\
& +(1-\lambda)^{2} f\left(\frac{a+b}{2}, \frac{c+d}{2}\right) \\
& +\frac{\lambda^{2}}{4}[f(a, c)+f(a, d)+f(b, c)+f(b, d)]-A \mid \\
& \leq(b-a)(d-c)\left(\frac{2 \lambda^{2}-2 \lambda+1}{4}\right)^{2-(2 / q)} \\
& \times\left(\iint_{0}^{1}|M(u, v)|\right. \\
& \times\left\{u v\left|\frac{\partial^{2} f}{\partial u \partial v}(a, c)\right|^{q}+u(1-v)\left|\frac{\partial^{2} f}{\partial u \partial v}(a, d)\right|^{q}\right. \\
& \left.\left.+(1-u)(1-v)\left|\frac{\partial^{2} f}{\partial u \partial v}(b, d)\right|^{q}\right\} d u d v\right)^{1 / q}
\end{aligned}
$$


On the other hand, we obtain

$$
\begin{aligned}
& \iint_{0}^{1}|M(u, v)| \\
& \times\left\{u v\left|\frac{\partial^{2} f}{\partial u \partial v}(a, c)\right|^{q}+u(1-v)\left|\frac{\partial^{2} f}{\partial u \partial v}(a, d)\right|^{q}\right. \\
& +(1-u) v\left|\frac{\partial^{2} f}{\partial u \partial v}(b, c)\right|^{q} \\
& \left.+(1-u)(1-v)\left|\frac{\partial^{2} f}{\partial u \partial v}(b, d)\right|^{q}\right\} d u d v \\
& =\left\{\left.\left|\frac{\partial^{2} f}{\partial u \partial v}(a, c)\right|\right|^{q} \iint_{0}^{1}|M(u, v)| u v d u d v\right. \\
& +\left|\frac{\partial^{2} f}{\partial u \partial v}(a, d)\right|^{q} \iint_{0}^{1}|M(u, v)| u(1-v) d u d v \\
& +\left|\frac{\partial^{2} f}{\partial u \partial v}(b, c)\right|^{q} \iint_{0}^{1}|M(u, v)|(1-u) v d u d v \\
& \left.+\left|\frac{\partial^{2} f}{\partial u \partial v}(b, d)\right| \iint_{0}^{1}|M(u, v)|(1-u)(1-v) d u d v\right\} \\
& =\left(\frac{2 \lambda^{2}-2 \lambda+1}{8}\right)^{2} \\
& \times\left(\left|\frac{\partial^{2} f}{\partial u \partial v}(a, c)\right|^{q}+\left|\frac{\partial^{2} f}{\partial u \partial v}(a, d)\right|^{q}\right. \\
& \left.+\left|\frac{\partial^{2} f}{\partial u \partial v}(b, c)\right|^{q}+\left|\frac{\partial^{2} f}{\partial u \partial v}(b, d)\right|^{q}\right) .
\end{aligned}
$$

Thus, we get the following inequality:

$$
\begin{aligned}
& \mid \frac{1}{(b-a)(d-c)} \int_{a}^{b} \int_{c}^{d} f(x, y) d y d x \\
& +(1-\lambda)^{2} f\left(\frac{a+b}{2}, \frac{c+d}{2}\right) \\
& +\frac{\lambda^{2}}{4}[f(a, c)+f(a, d)+f(b, c)+f(b, d)]-A \mid \\
& \leq(b-a)(d-c)\left(\frac{2 \lambda^{2}-2 \lambda+1}{4}\right)^{2} \\
& \times\left(\left(\left|\frac{\partial^{2} f}{\partial u \partial v}(a, c)\right|^{q}+\left|\frac{\partial^{2} f}{\partial u \partial v}(a, d)\right|^{q}\right.\right. \\
& \left.\left.+\left|\frac{\partial^{2} f}{\partial u \partial v}(b, c)\right|^{q}+\left|\frac{\partial^{2} f}{\partial u \partial v}(b, d)\right|^{q}\right) \times(4)^{-1}\right)^{1 / q},
\end{aligned}
$$

which completes the proof.
Remark 20. Applying Theorem 19 for $\lambda=0$, 1, we get the result of Theorems 6 and 10, respectively.

Theorem 21. Let $f: \Delta \subseteq \mathbb{R}^{2} \rightarrow \mathbb{R}$ be a partial differentiable mapping on $\Delta:=[a, b] \times[c, d]$ in $\mathbb{R}^{2}$ with $a<b$ and $c<d$. If $\left|\partial^{2} f / \partial u \partial v\right|^{q}$ is concave on the coordinates on $\Delta$ and $q>1$, then

$$
\begin{aligned}
& \mid \frac{1}{(b-a)(d-c)} \int_{a}^{b} \int_{c}^{d} f(x, y) d y d x \\
& +(1-\lambda)^{2} f\left(\frac{a+b}{2}, \frac{c+d}{2}\right)+\frac{\lambda^{2}}{4} \\
& \times[f(a, c)+f(a, d)+f(b, c)+f(b, d)]-A \mid \\
& \leq \frac{(b-a)(d-c)}{4}\left(\frac{\lambda^{p+1}+(1-\lambda)^{p+1}}{p+1}\right)^{2 / p} \\
& \quad \times\left|\frac{\partial^{2} f}{\partial u \partial v}\left(\frac{a+b}{2}, \frac{c+d}{2}\right)\right|,
\end{aligned}
$$

where $\lambda \in[0,1]$ and $A$ is as given in Theorem 15 and $1 / p+$ $1 / q=1$.

Proof. Similarly as in Theorem 17, because $\left|\partial^{2} f / \partial u \partial v\right|^{q}$ is a concave function on the coordinates on $\Delta$, by the reversed direction of (4), we get

$$
\begin{aligned}
& \iint_{0}^{1}\left|\frac{\partial^{2} f}{\partial u \partial v}(u a+(1-u) b, v c+(1-v) d)\right|^{q} d u d v \\
& \quad \leq\left|\frac{\partial^{2} f}{\partial u \partial v}\left(\frac{a+b}{2}, \frac{c+d}{2}\right)\right|^{q} .
\end{aligned}
$$

Hence, it follows that

$$
\begin{aligned}
& \mid \frac{1}{(b-a)(d-c)} \int_{a}^{b} \int_{c}^{d} f(x, y) d y d x \\
& +(1-\lambda)^{2} f\left(\frac{a+b}{2}, \frac{c+d}{2}\right)+\frac{\lambda^{2}}{4} \\
& \times[f(a, c)+f(a, d)+f(b, c)+f(b, d)]-A \mid \\
& \leq \frac{(b-a)(d-c)}{4}\left(\frac{\lambda^{p+1}+(1-\lambda)^{p+1}}{p+1}\right)^{2 / p} \\
& \quad \times\left|\frac{\partial^{2} f}{\partial u \partial v}\left(\frac{a+b}{2}, \frac{c+d}{2}\right)\right|
\end{aligned}
$$

which yields the desired result.

\section{Conflict of Interests}

The author has declared that no conflict of interests exists. 


\section{Acknowledgment}

This work is supported by Youth Project of Chongqing Three Gorges University of China (No. 13QN11).

\section{References}

[1] S. S. Dragomir, "Hermite-Hadamard's type inequalities for operator convex functions," Applied Mathematics and Computation, vol. 218, no. 3, pp. 766-772, 2011.

[2] S. S. Dragomir, "Hermite-Hadamard's type inequalities for convex functions of selfadjoint operators in Hilbert spaces," Linear Algebra and Its Applications, vol. 436, no. 5, pp. 1503-1515, 2012.

[3] A. El Farissi, "Simple proof and refinement of Hermite-Hadamard inequality," Journal of Mathematical Inequalities, vol. 4, no. 3, pp. 365-369, 2010.

[4] X. Gao, "A note on the Hermite-Hadamard inequality," Journal of Mathematical Inequalities, vol. 4, no. 4, pp. 587-591, 2010.

[5] S. S. Dragomir, "On the Hadamard's inequality for convex functions on the co-ordinates in a rectangle from the plane," Taiwanese Journal of Mathematics, vol. 5, no. 4, pp. 775-788, 2001.

[6] M. Alomari and M. Darus, "The Hadamard's inequality for $s$ convex function of 2-variables on the co-ordinates," International Journal of Mathematical Analysis, vol. 2, no. 13-16, pp. 629-638, 2008.

[7] M. A. Latif and M. Alomari, "On Hadmard-type inequalities for $h$-convex functions on the co-ordinates," International Journal of Mathematical Analysis, vol. 3, no. 33-36, pp. 1645-1656, 2009.

[8] M. Alomari and M. Darus, "On the Hadamard's inequality for log-convex functions on the coordinates," Journal of Inequalities and Applications, vol. 2009, Article ID 283147, 2009.

[9] M. A. Latif and S. S. Dragomir, "On some new inequalities for differentiable co-ordinated convex functions," Journal of Inequalities and Applications, vol. 2012, article 28, 2012.

[10] M. Z. Sarıkaya, E. Set, M. E. Ozdemir, and S. S. Dragomir, "New some Hadamard's type inequalities for co-ordinated convex functions," Tamsui Oxford Journal of Information and Mathematical Sciences, vol. 28, no. 2, pp. 137-152, 2012.

[11] M. E. Ozdemir, A. O. Akdemir, H. Kavurmaci, and M. Avci, "On the Simpson's inequality for co-ordinated convex functions," Classical Analysis and ODEs, http://arxiv.org/abs/1101.0075.

[12] M. E. Ozdemir, H. Kavurmaci, A. O. Akdemir, and M. Avci, "Inequalities for convex and s-convex functions on $\Delta=[a, b \times c, d]$," Journal of Inequalities and Applications, vol. 2012, article 20, 2012. 


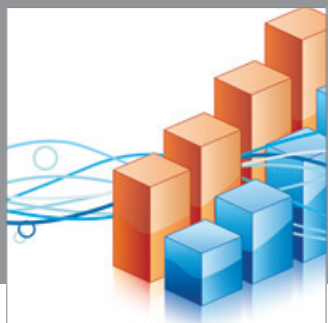

Advances in

Operations Research

mansans

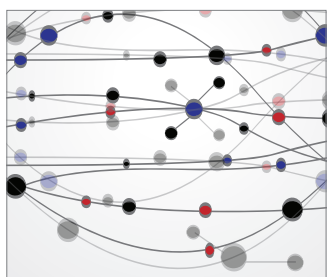

The Scientific World Journal
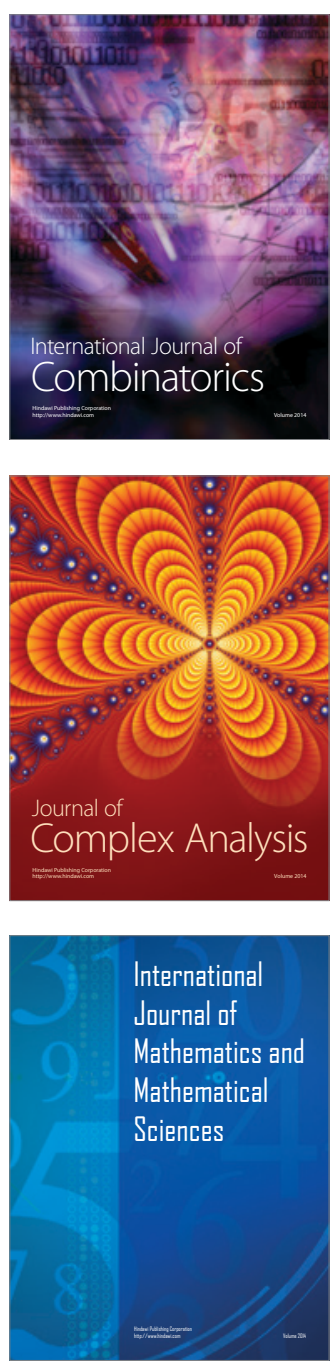
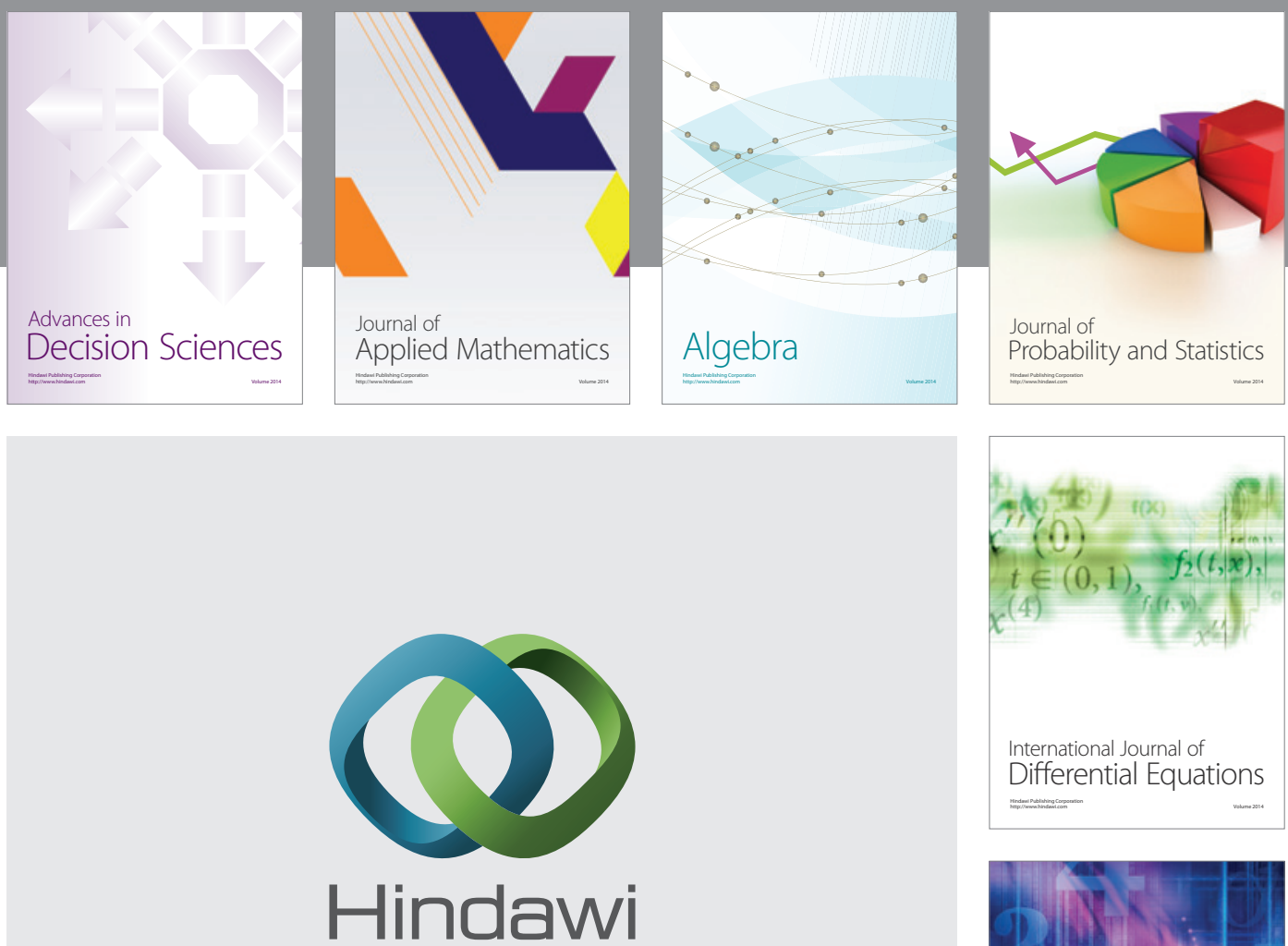

Submit your manuscripts at http://www.hindawi.com
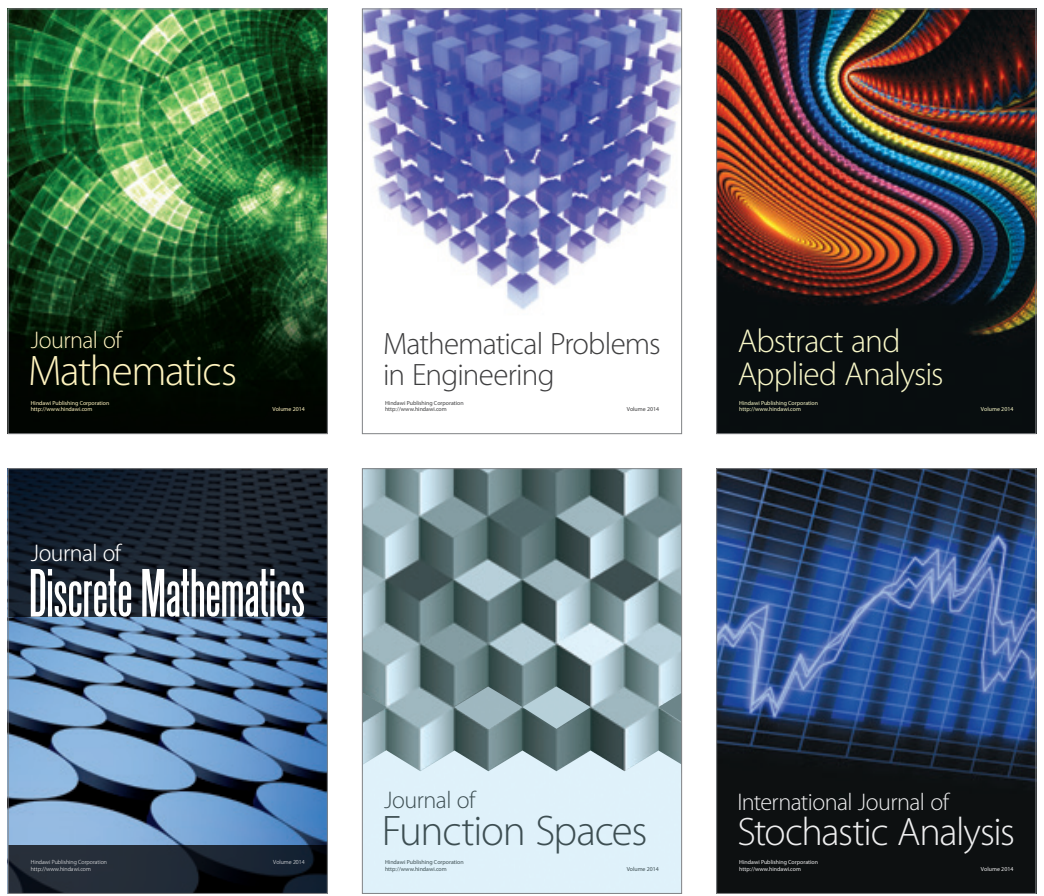

Journal of

Function Spaces

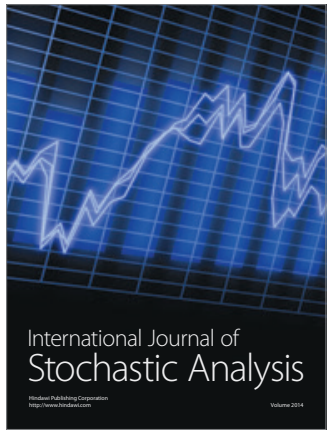

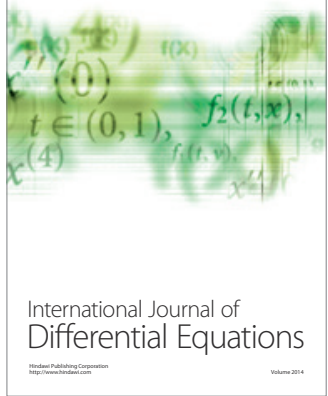
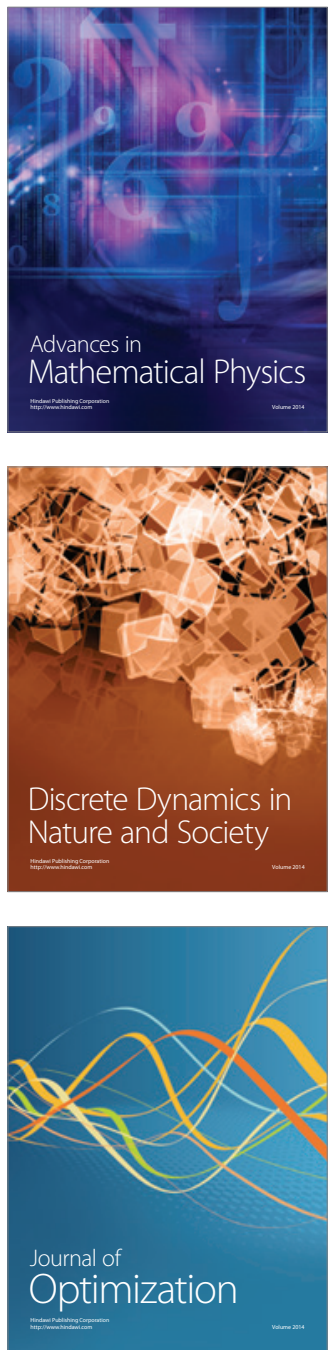